\title{
NMR and Neutron Scattering Investigation of Undercooled Aqueous Solutions of Apolar Solutes
}

\author{
S. Bradl and E. W. Lang* \\ Institut für Biophysik und Physikalische Biochemie, Universität Regensburg, \\ P.O. Box 101042, 93040 Regensburg, FRG
}

\section{J. Z. Turner}

Crystallography Department, Birkbeck College, London WC1E 7HX, U.K.

\author{
A. K. Soper \\ ISIS Science Division, Rutherford Appleton Laboratory, Oxon OX11 0QX, U.K. \\ Received: December 28, 1993; In Final Form: April 25, $1994^{\circ}$
}

\begin{abstract}
Multinuclear spin-lattice relaxation rates and self-diffusion coefficient measurements are reported over wide ranges of temperature, pressure, and concentration in undercooled aqueous solutions of tetramethylammonium bromide. These dissolved organic cations with apolar surface groups provide model systems to investigate the effect of Coulombic, hydrophobic, and $\mathrm{H}$-bond interactions upon the solvent and solute dynamics within the random, transient $\mathrm{H}$-bond network of the water. Also reported for the first time are neutron diffraction difference experiments in these undercooled solutions to investigate how changes in dynamic disorder are related to changes in the average static structure of the hydration water as temperature is decreased. A substantial sharpening of the orientational order between water molecules is observed in the undercooled state.
\end{abstract}

\section{Introduction}

The structure and the dynamic properties of a great number of aqueous solutions of atomic ions have been studied thoroughly both with neutron diffraction ${ }^{1}$ and nuclear magnetic relaxation ${ }^{2}$ techniques. Aqueous solutions with dissolved organic ions with apolar surface groups like symmetric tetraalkylammonium $\left(\mathrm{R}_{4} \mathrm{~N}^{+}\right)$ions are less well investigated. Despite many thermodynamic data, only very few investigations of the molecular dynamics in these systems exist ${ }^{3,4}$ and direct structural studies were reported only recently. 5,6

Aqueous solutions of $\mathbf{R}_{\mathbf{4}} \mathrm{N}^{+}$ions allow the competing influence of the Coulomb effect of the charge density and the hydrophobic effect of the apolar surface on the dynamic structure of the $\mathrm{H}$-bond network of water to be studied. Though the concept of hydrophobic hydration is widely used in chemistry and biology to describe the reduction in entropy upon solvation of apolar solutes in water, its molecular basis is not well understood yet. Thermodynamic, spectroscopic, ${ }^{3,4}$ and computer simulation 7,8 results suggest that the solvation of apolar groups (hydrophobic hydration) increases the amount of order in the random, transient $\mathrm{H}$-bond network of the coordinated water molecules and reduces their mobility. The hydration structures involved may resemble clathrate-like cages which would be favored by the network organization of liquid water. ${ }^{9}$

Further insight into the dynamic structure may be obtained in undercooled aqueous $\mathrm{R}_{4} \mathrm{~N}^{+}$solutions. Because of reduced thermal excitations hydrophobic hydration structures would become more stable and long-lived and hence would cause a strong slowing down of all molecular motions of all constituents. A recent report ${ }^{10}$ of the deuterium $\left({ }^{2} \mathrm{H}\right)$ spin-lattice relaxation rates (SLR) $R_{1}$ and self-diffusion coefficients $D_{\mathrm{s}}$ witnessed at ambient temperatures a slowing down of orientational and translational motions of hydration water molecules due to the interaction with the apolar surface and facilitated motions at low temperatures in the undercooled phase of aqueous tetramethylammonium bromide $\left(\mathrm{Me}_{4} \mathrm{NBr}\right)$ solutions. These data will be supplemented in this study by proton $\left({ }^{1} \mathrm{H}\right)$ and carbon- $13\left({ }^{13} \mathrm{C}\right)$ relaxation rate measurements providing insight into the overall and internal dynamics of the dissolved ions.

- Abstract published in Advance ACS Abstracts, July 1, 1994.

0022-3654/94/2098-8161\$04.50/0
These dynamical characteristics need to be confronted with direct structural information concerning the hydration shell around these apolar solutes. The latter is provided by partial pair correlation functions $g_{\alpha \beta}(r)$ as deduced from neutron diffraction experiments with isotope substitution (NDIS). The hydrogen-hydrogen correlation function $g_{\mathrm{HH}}(r)$ for the water in relatively concentrated aqueous solutions gives information on changes in the water structure caused by the presence of the solute relative to the structure in pure water. Previous measurements of the $\mathrm{HH}$-pair correlation function for water in tetramethylammonium chloride, $\mathrm{Me}_{4} \mathrm{NCl}$, at room temperature showed no appreciable change compared to pure water over a range of solute concentrations ${ }^{5,6}$ despite the fact that NMR measurements demonstrate an increased slowing down of the solvent mobility with increasing solute concentration at ambient temperatures. At low temperatures changes in the hydration water dynamics occur with facilitated molecular motions in the solutions compared to pure water in the deeply undercooled state.

Motivated by these complex changes in the dynamics of the hydration water in undercooled solutions as revealed by our recent NMR experiments, ${ }^{10}$ we report in this study for the first time neutron diffraction experiments with $H / D$ substitution in the water in undercooled aqueous TMABr solutions. The neutron diffraction results presented in this work are a first step of an investigation into whether such changes in dynamic disorder are related to changes in the average static structure of the water in solutions of tetraalkylammonium ions as temperature is decreased.

\section{Experiment}

NMR Experiments. Tetramethylammonium bromide $\left(\mathrm{Me}_{4}-\right.$ $\mathrm{NBr}$ ) was purchased from Aldrich Chemicals (Steinheim, FRG) and heavy water $\left(\mathrm{D}_{2} \mathrm{O}, 99.8\right.$ at. \%) was obtained from Merck (Darmstadt, FRG). The salt was dried on a vacuum line for 24 $\mathrm{h}$, and the solutions were prepared by weighing the proper amount of salt to $3 \mathrm{~mL}$ of heavy water. Afterwards the solutions were degassed on a vacuum line by at least five freeze-pump-thaw cycles and then filled into the high-pressure glass capillary as described elsewhere. ${ }^{11,12}$ To reach high degrees of undercooling, emulsions had to be used, however. As supporting alkane phase, deuterated methylcyclohexane ( $\mathrm{MCH}-d_{14}$, Riedel-de Haen, 
Seelze, FRG) and a small amount of the surfactant sorbitane tristearate (Span 65, Merck) were used. Emulsions were then produced under an argon atmosphere in a glovebag (AtmosBag, Aldrich Chemicals) by pressing the alkane/surfactant/aqueous solution mixture through a stainless steel net (Spörl \& Co., Sigmaringendorf, FRG) within a syringe. Afterwards the emulsion was filled into the high-pressure capillaries on the vacuum line. All experiments were performed on a Bruker MSL 300 multipurpose solid-liquid NMR spectrometer equipped with a variable temperature unit VT 100 and a pulsed magnetic field gradient unit BZ 18B. The probes for the SLR measurements consisted of home-built $10-\mathrm{mm}$ probe heads with an external ${ }^{2} \mathrm{H}$ lock suitable for operation in the ranges $280-300 \mathrm{MHz}$ for ${ }^{1} \mathrm{H}$ and $70-80 \mathrm{MHz}$ for ${ }^{13} \mathrm{C}$ nuclei, respectively. An additional radio frequency coil (rf-coil) tuned to $300 \mathrm{MHz}$ in the latter probe head served the needs for broad-band decoupling experiments. The SLR times $T_{1}$ were determined with nonselective pulses with the inversion-recovery pulse sequence with alternating phases. Data were then analyzed with a three parameter nonlinear least squares regression program. The relaxation times are considered reliable to within $\pm 10 \%$.

The temperature was varied with a flow of cold nitrogen gas appropriately heated and regulated by the VT 100 unit. It was measured at the center of the rf-coil with a chromel-alumel thermocouple (Philips, Kassel, FRG) before and after each series of inversion-recovery experiments. The temperatures are reliable to $\pm 1 \mathrm{~K}$. The pressure was generated with a home-built highpressure apparatus suitable for operation up to $400 \mathrm{MPa}$ and was measured with a Bourdon gauge (Heise, Connecticut) with a precision of $\pm 0.5 \mathrm{MPa}$.

NDIS Experiments. Diffraction measurements were made on three samples of deuterated $\mathrm{Me}_{4} \mathrm{NBr}$ at a concentration of 1:27 water molecules $(2 \mathrm{~m})$ corresponding to approximately one independent water layer around each cation. The deuterated salt (98\% D) was purchased by IC Chemikalien (Ismaning, FRG), dried under vacuum for $24 \mathrm{~h}$, and used without further purification. The three solutions were made up in (a) $\mathrm{D}_{2} \mathrm{O}$, (b) $\mathrm{H}_{2} \mathrm{O}$, and (c) a null mixture of 0.64 mole fraction $\mathrm{H}_{2} \mathrm{O}$ and 0.36 mole fraction $\mathrm{D}_{2} \mathrm{O}$ for which the coherent scattering of the protons and deuterons cancels. The measurements were made at room temperature and at $258 \mathrm{~K}$ using the standard closed cycle refrigerator (CCR) available at ISIS. To avoid crystallization, the solutions had to be filled into precision-made quartz capillaries (Westdeutsche Quartzschmelze, Geesthacht, FRG) with an o.d. of $1 \mathrm{~mm}$ and an i.d. of $0.7 \mathrm{~mm}$. The solutions were first degassed by several freezepump-thaw cycles on a vacuum line and then filled into the evacuated capillaries. The latter were sealed on one end and immersed into the solution after evacuation. The solution was then forced into the capillaries by applying argon gas pressure onto the liquid surface within the filling device on the vacuum line. The capillaries were then removed and flame-sealed. Afterwards their vacuum tightness was tested for $24 \mathrm{~h}$ on a vacuum line. Furthermore they had to be tested for their supercooling ability by immersing the capillaries in a heat bath into liquid ethanol, the temperature of which was regulated to $258 \pm 1 \mathrm{~K}$ for $48 \mathrm{~h}$ at least. The freezing of the capillaries was checked under a microscope.

The capillaries were then placed in rows into a specially designed sample holder (see Figure 1) which consisted of a mounting device and a removable frame, both made of copper to ensure a good thermal contact between the CCR and the quartz capillaries. The latter were inserted from top into narrow bores in the upper and lower part of the $\mathrm{Cu}$-frame. A movable grid with exactly the same holes in exactly corresponding positions helped to fix the quartz tubes in their exact position within the beam. One row (30 holes with diameter $d=1.2 \mathrm{~mm}$ and a center-center distance of $1.3 \mathrm{~mm}$ ) was used for the hydrogeneous samples and two rows for the $\mathrm{D}_{2} \mathrm{O}$ sample. The row of capillaries spanned the beam width and was treated in the analysis as flat plate geometry. During the measurements at $258 \mathrm{~K}$ possible crystallization was

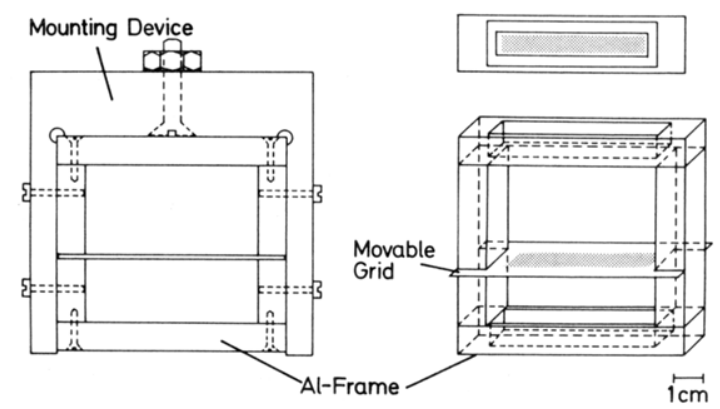

Figure 1. Sample holder for NDIS experiments.

monitored by checking for the presence of Bragg peaks over a wide $Q$ range.

The diffraction experiments were made on the SANDALS time-of-flight diffractometer at the ISIS neutron scattering facility. ${ }^{13}$ The advantage of using this diffractometer for hydrogeneous liquid samples is that inelasticity corrections are minimized by the use of small scattering angles and high incident neutron energies, while the diffraction measurements can be made up to high values of the scattering vector. The useful range in scattering vector $\mathbf{Q}$ obtained and used in the calculation of the partial structure factors was $0.5-30 \AA^{-1}$, where $Q=4 \pi \sin \theta / \lambda$; the scattering angle is $2 \theta$, and $\lambda$ is the neutron wave length. Diffraction measurements were also made on the empty quartz tubes and on a vanadium slab of dimensions $\times 50 \times 50 \times 3.2 \mathrm{~mm}$. The counting time was between 12 and $24 \mathrm{~h} /$ sample. The diffraction data from approximately 460 detectors were converted to a common $Q$ scale and divided into six groups corresponding to scattering angles between 11 and 20 degrees. The data for each group of detectors were normalized by the incident monitor count and corrected for absorption and multiple scattering in the sample and quartz tubes using the ATLAS series of programms. ${ }^{14}$ The differential scattering cross-section per atom, $\Sigma_{\mathrm{T}}(Q, 2 \theta)$, was then calculated for each group of detectors from the corrected diffraction intensity by reference to the scattering from the vanadium slab. The single atom scattering was then subtracted from the differential scattering cross-section, as is described in detail in ref 6, to obtain the interference function for each detector group. The data from each group were then combined to form a single interference function $F(Q)$.

\section{NMR Spin-Lattice Relaxation Rates and Self-Diffusion Coefficients}

Deuterium Spin-Lattice Relaxation Rates. Deuterium spinlattice relaxation (SLR) rates $R_{1}\left({ }^{2} \mathrm{H}\right)$ of the solvent deuterons have been measured as a function of pressure ( $p \leq 225 \mathrm{MPa})$, temperature $(230 \mathrm{~K} \leq T \leq 360 \mathrm{~K})$, and concentration $(c=1$, $2,3 \mathrm{~m})$. These results have been reported recently ${ }^{10}$ in connection with similar experiments on related undercooled aqueous tetraalkylammonium halide solutions, and a thorough discussion of the solvent dynamics in these solutions has been given.

According to published hydration number estimates, ${ }^{15}$ a $2 \mathrm{~m}$ solution corresponds to a composition (1:25) where all water molecules are directly coordinated to the dissolved $\mathrm{Me}_{4} \mathrm{~N}^{+}$cations. Their diffusion coefficient may be considered to represent the dynamics of the hydration water molecules. Deuterium SLR times $\left(T_{1}\left({ }^{2} \mathrm{H}\right)\right)$ monitor reorientational motions of water molecules which in the fast motions regime $(\omega \tau<1)$ are proportional to their average rotational diffusion coefficient, i.e. $T_{1}\left({ }^{2} \mathrm{H}\right) \approx D_{\mathrm{r}}$. The relaxation times exhibit an isobaric temperature dependence which is neither observed in the case of strongly hydrating (i.e. $\mathrm{Li}^{+}$) nor weakly hydrating (i.e. $\mathrm{Cs}^{+}$) atomic ions, as is demonstrated in Figure 2a which compares the $T_{1}\left({ }^{2} \mathrm{H}\right)$ of water molecules directly coordinated to $\mathrm{Li}^{+}, \mathrm{Cs}^{+}$, and $\left(\mathrm{CH}_{3}\right)_{4} \mathrm{~N}^{+}$with the relaxation in pure $\mathrm{D}_{2} \mathrm{O}^{2}$ It is seen that if the $\mathrm{H}$-bond network is strongly perturbed because of thermal excitations, these large apolar ions reduce the average mobility of the water molecules. At low temperatures, however, $\mathrm{H}$-bond interactions in the random, 

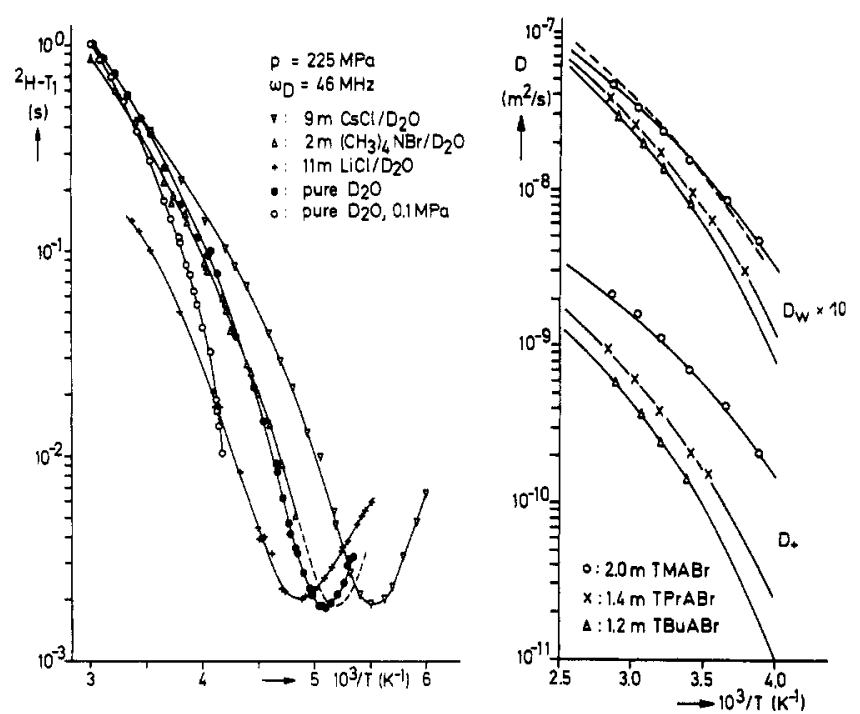

Figure 2. (a) Comparison of the spin-lattice relaxation times $\left(T_{1}\left({ }^{2} \mathrm{H}\right)\right)$ of the hydration water molecules in undercooled $\mathrm{LiCl} / \mathrm{D}_{2} \mathrm{O}(+, 11 \mathrm{~m})$, $\mathrm{CsCl} / \mathrm{D}_{2} \mathrm{O}(\nabla, 9 \mathrm{~m})$, and $\mathrm{Me}_{4} \mathrm{NBr} / \mathrm{D}_{2} \mathrm{O}(\Delta, 2 \mathrm{~m})$ solutions and pure heavy water $(0,0.1 \mathrm{MPa} ; \bullet, 225 \mathrm{MPa})$. (b) Comparison of the selfdiffusion coefficients of the hydration water molecules $D_{w}$ in undercooled (O) $\mathrm{Me}_{4} \mathrm{NBr} / \mathrm{D}_{2} \mathrm{O}$, (x) $\mathrm{Pr}{ }_{4} \mathrm{NBr} / \mathrm{D}_{2} \mathrm{O}$, and $(\Delta) \mathrm{Bu} 4 \mathrm{NBr} / \mathrm{D}_{2} \mathrm{O}$ solutions and pure water (-.). Corresponding cationic diffusion coefficients $D_{+}$ are also shown.

transient $\mathrm{H}$-bond network of pure water slow down reorientational fluctuations stronger than ion-water interactions. Accordingly, the apolar surface of the dissolved ions distorts water-water interactions and the average mobility of the hydration water molecules is enhanced compared to pure water. This behavior is even observed in solutions under high pressure, which strongly perturbs $\mathrm{H}$-bond interactions, too. There strongly hydrating atomic ions with a high charge density reduce the water mobility at all temperatures and weakly hydrating ions with a small charge density always increase the latter. Therefore, as the charge density is even smaller in $\mathrm{Me}_{4} \mathrm{~N}^{+}$ions, the reduced solvent mobility observed at ambient temperatures can result only from increased $\mathrm{H}$-bond interactions between the hydration water molecules, which are induced by the apolar surface. The latter is supposed to impose an orientational bias whereby the adjacent water molecules orient themselves in a way so as to maximize their $\mathrm{H}$-bonding interactions. If, however, $\mathrm{Me}_{4} \mathrm{~N}^{+}$ions were to induce clathratelike transient structures which are believed to provide dominant structural features in the H-bond network of undercooled water, ${ }^{9}$ then a continued further reduction of the average mobility down to the lowest temperatures might be expected. This has not been observed, however, and indicates the subtle balance between Coulombic, hydrophobic hydration, and $\mathrm{H}$-bond interactions in these solutions.

Self-Diffusion Coefficients. Similar conclusions can be drawn from the temperature dependence of the self-diffusion coefficient $D_{w}$ of the hydration water molecules, as shown in Figure $2 \mathrm{~b}$. Compared to the translational mobility of water molecules in the random, transient $\mathrm{H}$-bond network of pure water, hydration water molecules show a reduced mobility at high temperatures, corroborating earlier room temperature data ${ }^{15-17}$ but facilitated diffusion in the undercooled phase. Again the latter result would not have been expected if hydration water molecules would be organized into transient clathrate-like structures with strengthened $\mathrm{H}$-bonds around the apolar surface of the $\mathrm{Me}_{4} \mathrm{~N}^{+}$cations. Indeed dissolved organic cations with larger apolar surface groups, like $\left(\mathrm{C}_{3} \mathrm{H}_{7}\right)_{4} \mathrm{~N}^{+}$and $\left(\mathrm{C}_{4} \mathrm{H}_{9}\right)_{4} \mathrm{~N}^{+}$, reduce the translational mobility of the respective hydration water molecules considerably, as is seen in Figure 2b.

In this respect it is also noteworthy that the diffusion coefficient of the hydration water molecules is larger than the diffusion coefficient of the $\mathrm{Me}_{4} \mathrm{~N}^{+}$ions. This implies that the lifetime $\tau_{\mathrm{ex}}$ of a water molecule in the hydration shell of these apolar cations
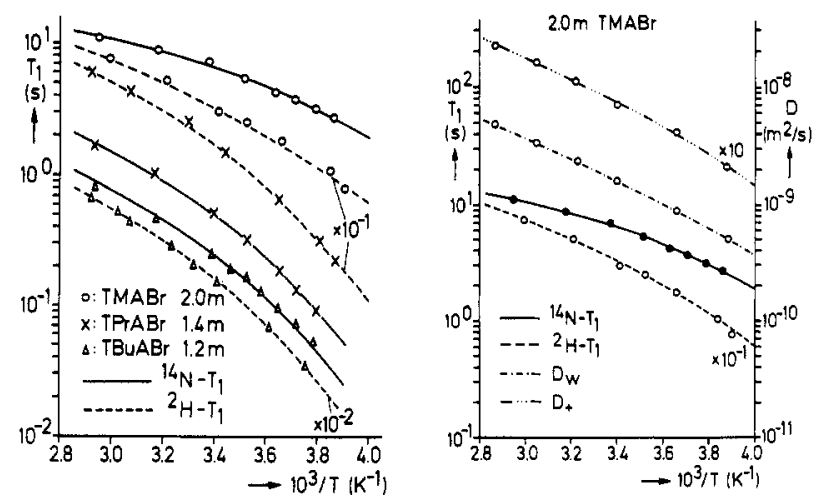

Figure 3. (a) Comparison of the deuterium $\left({ }^{2} \mathrm{H}\right)$ and nitrogen-14 $\left({ }^{14} \mathrm{~N}\right)$ spin-lattice relaxation times in undercooled aqueous $\mathrm{Me}{ }_{4} \mathrm{NBr}(2 \mathrm{~m})$, $\mathrm{Pr}_{4} \mathrm{NBr}(1.4 \mathrm{~m})$, and $\mathrm{Bu}_{4} \mathrm{NBr}(1.2 \mathrm{~m})$ solutions. At the given concentrations all water molecules are coordinated to the dissolved organic cations. (b) Comparison of the isobaric temperature dependence of $T_{1}\left({ }^{14} \mathrm{~N}\right)$ of the $\mathrm{Me}_{4} \mathrm{~N}^{+}$ions, $T_{1}\left({ }^{2} \mathrm{H}\right)$ of the hydration water molecules, and the respective self-diffusion coefficients $D_{+}$and $D_{\mathrm{w}}$ for a $2 \mathrm{~m}$ undercooled $\mathrm{Me}_{4} \mathrm{NBr} / \mathrm{D}_{2} \mathrm{O}$ solution.

cannot exceed the translational correlation time $\tau_{+, \text {pos }}=\left\langle\boldsymbol{R}_{+}{ }^{2}\right\rangle /$ $2 D_{+}$characterizing the diffusive motions of the solute, i.e. $\tau_{\mathrm{ex}}<$ $\tau_{+, \text {pos }}{ }^{13}$ Both observations corroborate the conclusions drawn from the interpretation of the rotational dynamics in that characteristics of electrostatic hydration and hydrophobic hydration are largely balanced and that the hydration water dynamics depend strongly on the state of the H-bond network.

Nitrogen-14 Spin-Lattice Relaxation Rates. Nitrogen-14 spinlattice relaxation times of the dissolved $\mathrm{Me}_{4} \mathrm{~N}^{+}$ions probe fluctuations of the electric field gradient (efg) at the central ${ }^{14} \mathrm{~N}$ nucleus due to symmetry fluctuations of the hydration shell and molecular motions of the constituent water molecules and dissolved ions. According to the electrostatic theory of quadrupolar relaxation, ${ }^{18}$ the relaxation rate is given by $\left({ }^{14} \mathrm{~N}: I=1\right)$

$$
\begin{array}{r}
R_{1, Q}\left({ }^{14} \mathrm{~N}\right)=6 \pi\left[(2 \pi \mathrm{e} Q / h)\left(1+\gamma_{\infty}\right) P\right]^{2}\left[\left(m^{2} / r_{\mathrm{o}}{ }^{5}\right) c_{\mathrm{w}} \tau_{\text {or }, \mathrm{w}}+\right. \\
\left.\sum\left(z_{j} / 3 a_{j}\right) c_{j} \tau_{\mathrm{pos}, j}\right]
\end{array}
$$

with $m$ the electric dipole moment of the water, $c_{w}$ and $c_{j}$ the solvent and solute concentrations, $r_{0}$ and $a_{j}$ the distances of closest approach of a water molecule and another ion, respectively, $\tau_{\text {or,w }}$ the rotational correlation time of the coordinated water molecules, $\tau_{\text {pos } j}$ the transitional correlation time of anions or cations, $\gamma_{\infty}$ the Sternheimer antishielding factor, and $P$ a polarization factor. Hence, the $T_{1}\left({ }^{14} \mathrm{~N}\right)$ of the $\mathrm{Me}_{4} \mathrm{~N}^{+}$cations should be proportional to the rotational diffusion coefficient $D_{\mathrm{r}} \approx 1 / \tau_{\mathrm{or}, \mathrm{w}}$, respectively, the $T_{1}\left({ }^{2} \mathrm{H}\right)$, of the hydration water molecules, and the ionic selfdiffusion coefficients $D_{+} \approx 1 / \tau_{\text {pos } j .}$. The latter could not be measured for the bromide anions; hence a quantitative evaluation cannot be given. A qualitative comparison of the temperature dependence of the nitrogen-14 and the deuterium relaxation times of the organic ions and the $\mathrm{D}_{2} \mathrm{O}$ molecules in the undercooled aqueous solutions of $\mathrm{Me}_{4} \mathrm{NBr}(2 \mathrm{~m}), \mathrm{Pr}_{4} \mathrm{NBr}(1.4 \mathrm{~m})$, and $\mathrm{Bu}_{4}$ $\mathrm{NBr}(1.2 \mathrm{~m})$ is made in Figure 3a. Both relaxation times show a parallel temperature dependence in the systems $\operatorname{Pr}_{4} \mathrm{~N}^{+}-\mathrm{D}_{2} \mathrm{O}$ and $\mathrm{Bu}_{4} \mathrm{~N}^{+}-\mathrm{D}_{2} \mathrm{O}$, suggesting that the orientational fluctuations of the hydration water dipoles dominate the relaxation of the ${ }^{14} \mathrm{~N}$ spins in these solutions. The situation is more complex, however, in the $\mathrm{Me}_{4} \mathrm{~N}^{+}-\mathrm{D}_{2} \mathrm{O}$ solutions, as is evident from Figure $3 \mathrm{~b}$. Neither the reorientation of the hydration water dipoles nor their translational diffusion or one of the other cations really dominates the relaxation of the ${ }^{14} \mathrm{~N}$ nuclei of the cations. This is a further indication of the complicated solvent dynamics prevailing in these solutions due to the variety of forces embodied including hydrogen bonding and electrostatic and hydrophobic interactions.

Proton and Carbon-13Spin-Lattice Relaxation Rates. Further insight into the dynamics of the dissolved cations may be obtained from ${ }^{1} \mathrm{H}$ and ${ }^{13} \mathrm{C}$ spin-lattice relaxation rates. They have been 

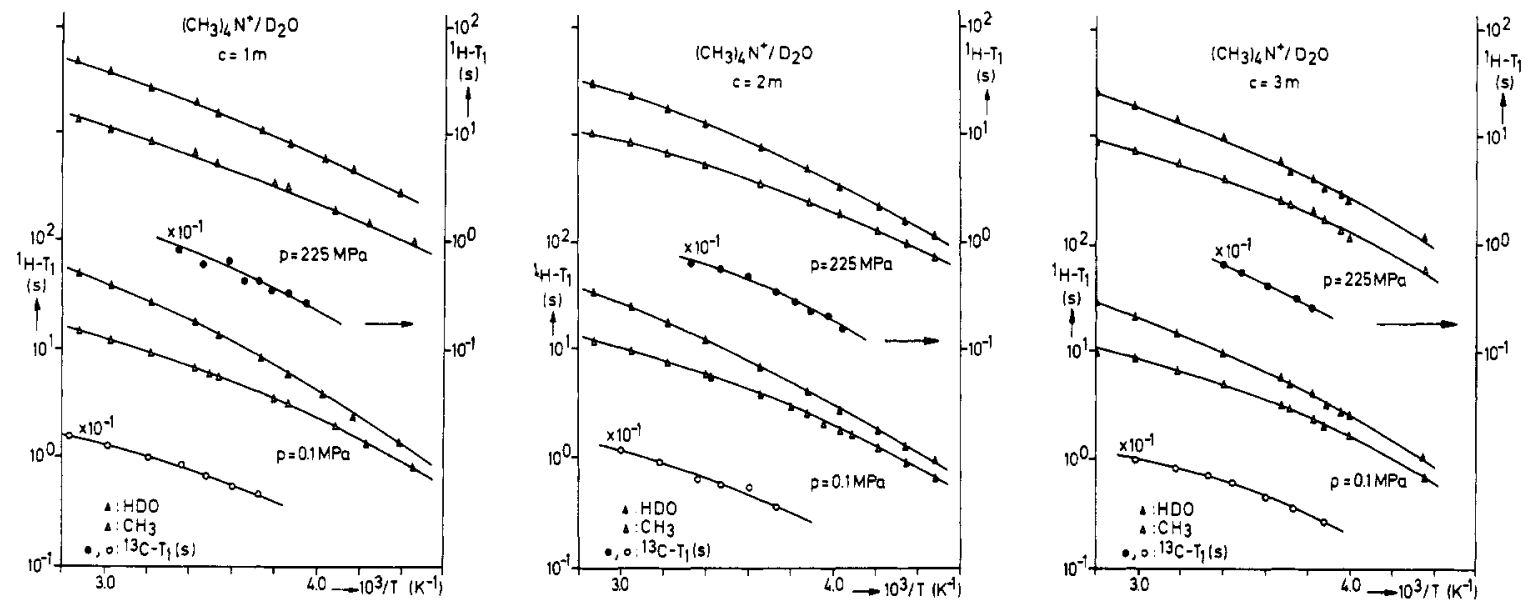

Figure 4. Isobaric temperature dependence of the proton $\left({ }^{1} \mathrm{H}\right)$ and carbon-13 $\left({ }^{13} \mathrm{C}\right)$ spin-lattice relaxation times of the $\mathrm{Me}_{4} \mathrm{~N}^{+}$ions and the solvent (HDO) molecules at the concentrations (a) 1.0, (b) 2.0, and (c) $3.0 \mathrm{~m}$.

determińed over a large temperature $(220 \mathrm{~K}<T<350 \mathrm{~K})$, pressure ( $p \leq 225 \mathrm{MPa})$, and concentration $(c=1,2,3 \mathrm{~m})$ range. Figure 4 shows the temperature dependence of all relaxation times measured at $p=0.1$ and $225 \mathrm{MPa}$, respectively, in solutions of $\left(\mathrm{CH}_{3}\right)_{4} \mathrm{NBr}$ in water $\left(5 \% \mathrm{H}_{2} \mathrm{O}, 95 \% \mathrm{D}_{2} \mathrm{O}\right)$ at the concentrations given. Again a $2 \mathrm{~m}$ solution corresponds to a composition where all solvent molecules are nearest neighbors to the apolar cations.

Spin-lattice relaxation rates of carbon-1 3 nuclei are dominated by dipolar couplings to $n_{\mathrm{H}}$ directly bonded protons and are given by ${ }^{19}$

$$
\begin{array}{r}
R_{1, \mathrm{OR}}\left({ }^{13} \mathrm{C}-{ }^{1} \mathrm{H}\right)=n_{\mathrm{H}} C_{\mathrm{or}}(\mathrm{C}, \mathrm{H})\left(g\left(\omega_{\mathrm{C}}-\omega_{\mathrm{H}}\right)+3 g\left(\omega_{\mathrm{C}}\right)+\right. \\
\left.6 g\left(\omega_{\mathrm{C}}+\omega_{\mathrm{H}}\right)\right)
\end{array}
$$

with

$$
C_{\mathrm{or}}(\mathrm{C}, \mathrm{H})=(2 / 15)\left(\gamma_{\mathrm{H}} \gamma_{\mathrm{C}} \hbar \mu_{0} / 4 \pi\right)^{2} S(S+1)
$$

and with $\gamma_{\mathbf{H}}, \gamma_{\mathbf{C}}$ the gyromagnetic ratio of the protons and carbon13 nuclei, respectively, and with $S=1 / 2$ the spin quantum number of the protons. The other symbols have their usual meaning. The spectral density $g(\omega)$ may be evaluated within the "model-free" approach of Lipari and Szabo. ${ }^{20}$ The corresponding functions $g_{\text {or }}(\omega)$ are given by ${ }^{20}$

$$
\begin{aligned}
& g_{\text {or }}(\omega)=S^{2}\left(\tau_{+, \mathrm{or}} /\left(1+\left(\omega \tau_{+, \mathrm{or}}\right)^{2}\right)\right)+ \\
&\left(1-S^{2}\right)\left(\tau /\left(1+(\omega \tau)^{2}\right)\right)\left\langle 1 / R_{\mathrm{CH}}{ }^{3}\right)^{2}
\end{aligned}
$$

where $1 / \tau=1 / \tau_{+, \text {or }}+1 / \tau_{\text {int }}$ with $\tau_{+, \text {or }}$ the correlation time of the overall tumbling motion of the organic cations and $\tau_{\text {int }}$ the correlation time of their internal methyl group reorientation. The generalized order parameter $S^{2}$ provides a measure of the spatial restriction of this local mode. In case of a methyl group reorientation around the $\mathrm{C}-\mathrm{N}$ axis, one obtains

$$
S^{2}=(1 / 2)\left(3 \cos ^{2} \theta-1\right)= \begin{cases}0.11, & \text { C-H vector } \\ 0.25, & \text { H-H vector }\end{cases}
$$

If time scale separation prevails between both motional modes, i.e. $\tau_{+, \text {or }} \gg \tau_{\text {int }}$, then the above expression reduces to the two-step model of Halle and Wennerström. ${ }^{21}$ In the fast motions limit $(\omega \tau \ll 1)$ the above expression, eq 3 , simplifies to

$$
g_{\text {or }}(\omega)=\left(S^{2} \tau_{+, \text {or }}+\left(1-S^{2}\right) \tau\right)\left(1 / R_{C H}{ }^{3}\right\rangle^{2}
$$

Proton spin-lattice relaxation rates may be decomposed into a sum of intra- and intermolecular contributions. The intermolecular contribution $R_{1, p o s}$ is mainly due to dipolar interactions with protons on other cations as the solvent is fully deuterated. This interaction is modulated by relative translational motions
TABLE 1: Densities of $2 m$ Tetramethylammonium Bromide (a) and Chloride (b) Solutions in $\mathrm{H}_{2} \mathrm{O}$ and $\mathrm{D}_{2} \mathrm{O}$, Respectively"

\begin{tabular}{lrr}
\hline & \multicolumn{2}{c}{ densities $\left(\mathrm{kg} / \mathrm{m}^{3}\right)$} \\
\cline { 2 - 3 }$T(\mathrm{~K})$ & \multicolumn{1}{c}{$\mathrm{H}_{2} \mathrm{O}$} & \multicolumn{1}{c}{$\mathrm{D}_{2} \mathrm{O}$} \\
\hline 308.4 & 1057.542 & $1144.272(\mathrm{a})$ \\
& 997.147 & $1085.876(\mathrm{~b})$ \\
297.4 & 1062.035 & $1148.277(\mathrm{a})$ \\
& 1000.783 & $1089.570(\mathrm{~b})$ \\
288.3 & 1063.031 & $1148.772(\mathrm{a})$ \\
& 1001.496 & $1089.871(\mathrm{~b})$ \\
278.0 & 1067.980 & $1153.608(\mathrm{a})$ \\
& 1005.955 & $1094.155(\mathrm{~b})$ \\
$258.0^{*}$ & 1073.991 & $1158.748(\mathrm{a})$ \\
& 1010.872 & $1098.708(\mathrm{~b})$
\end{tabular}

a The data at $258 \mathrm{~K}$ have been obtained by extrapolation.

of the $\mathrm{Me}_{4} \mathrm{~N}^{+}$ions. A large distance of closest approach $d_{\mathrm{HH}}$ of the hydrated cations, and slow motions render this relaxation path of minor importance, however. It may be calculated within a diffusion model with reflecting boundary conditions at the distance of closest approach $d_{\mathrm{HH}} \cdot{ }^{11,12}$ Any structure in the radial pair distribution function $g_{\mathrm{HH}}(r)$ was neglected, however. The number density of the protons can be calculated from the experimental mass density, which has been determined for a few temperatures with a mechanical oscillator technique ${ }^{22}$ and is tabulated in Table 1. The contribution of $R_{1, \text { pos }}$ to the total proton relaxation rate $R_{1}\left({ }^{1} \mathrm{H}\right)$ is small in all cases considered. ${ }^{23}$ The latter is dominated by the intramolecular contribution $R_{1, \text { or }}$ which is related to the dipolar coupling of all protons located on the same molecule. These interactions fluctuate due to the overall tumbling of the whole cation as well as the local anisotropic methyl group reorientation. The corresponding relaxation rate between a nuclear spin $I$ and spins $I^{\prime}$ is given in general by ${ }^{19}$

$$
\begin{array}{r}
R_{1, \mathrm{or}}\left(\mathrm{I}, \mathrm{I}^{\prime}\right)=\Sigma_{\mathrm{I}^{\prime}}(2 / 5)\left(\gamma_{\mathrm{H}}{ }^{2} \hbar \mu_{0} / 4 \pi\right) \mathrm{I}^{\prime}\left(\mathrm{I}^{\prime}+1\right)\left[g_{\text {or }}\left(\omega_{\mathrm{H}}\right)+\right. \\
\left.4 g_{\text {or }}\left(2 \omega_{\mathrm{H}}\right)\right]
\end{array}
$$

and the appropriate spectral density functions are given by eq 5 with $R_{\mathrm{HH}^{\prime}}$ replacing $R_{\mathrm{CH}}$. Within a rigid molecule approximation ${ }^{24}$ the corresponding intramolecular internuclear distances $R_{\mathrm{HH}^{\prime}}$ are given in Table 2 . The possibly small effect of a variation of the intermethyl group interactions due to methyl group reorientations has been neglected in this summation of the dipolar interactions. Anyhow, due to the $R^{-6}$ dependence on internuclear separation, the dipolar coupling within any given methyl group dominates the intramolecular proton relaxation, hence the total proton relaxation rate $R_{1}\left({ }^{1} \mathrm{H}\right)$ also.

Correlation Times. Given the order parameter $S^{2}$ and the relevant internuclear distances $R_{\mathrm{IS}}$, the main objective remains the determination of the correlation times $\tau_{+, \text {or }}$ and $\tau_{\text {int }}$ and their dependence on the external variables $(T, p, c)$ from a simultaneous 
TABLE 2: Intramolecular Distances $(\AA)$ between the Methyl Group Protons in the $\left(\mathrm{CH}_{3}\right)_{4} \mathrm{~N}^{+}$Ion

\begin{tabular}{ccccc}
\hline & & $\mathrm{H}_{1}$ & $\mathrm{H}_{2}$ & $\mathrm{H}_{3}$ \\
\hline $\mathrm{X}$ & $\mathrm{H}_{1}$ & & 1.782 & 1.782 \\
& $\mathrm{H}_{2}$ & 1.782 & & 1.782 \\
$-\mathrm{X}$ & $\mathrm{H}_{3}$ & 1.782 & 1.782 & \\
& $\mathrm{H}_{1}$ & 4.182 & 3.640 & 3.640 \\
& $\mathrm{H}_{2}$ & 3.640 & 2.991 & 2.408 \\
$\mathrm{Y}$ & $\mathrm{H}_{3}$ & 3.640 & 2.408 & 2.991 \\
& $\mathrm{H}_{1}$ & 2.991 & 3.643 & 2.419 \\
& $\mathrm{H}_{2}$ & 3.643 & 3.657 & 4.201 \\
$-\mathrm{Y}$ & $\mathrm{H}_{3}$ & 2.419 & 3.017 & 3.657 \\
& $\mathrm{H}_{1}$ & 2.991 & 2.419 & 3.643 \\
& $\mathrm{H}_{2}$ & 2.419 & 3.657 & 3.017 \\
& $\mathrm{H}_{3}$ & 3.643 & 4.201 & 3.657
\end{tabular}

fit to the proton and carbon- 13 relaxation rates. According to hydrodynamic theory the overall tumbling correlation times $\tau_{+, \text {or }}$ of the large and nearly spherical cations may be related to the ionic self-diffusion coefficient via the Debye-Stokes-Einstein relation ${ }^{25}$ yielding

$$
\tau_{+, \mathrm{or}}(T, p, c)=(4 / 3 C)\left(R_{\mathrm{eff}}^{2} / D_{+}(T, p, c)\right)
$$

with $C=6$ or 4 a parameter characterizing stick or slip boundary conditions on the surface of the apolar solute, $R_{\text {eff }}$ an effective hydrodynamic radius approximating the ion as a spherical particle, and $D_{+}$the experimental self-diffusion coefficient of the cations. The isobaric temperature dependence of the latter can be reproduced well ${ }^{10}$ with a modified Arrhenius equation

$$
D_{+}(T, p, c)=D_{0} \exp \left(B_{+, \mathrm{pos}} /\left(T-T_{0}(c, p)\right)\right)
$$

with $T_{0}$ a concentration and pressure dependent kinetic localization temperature, often called the ideal glass transition temperature. The latter has been determined from a consistent description of deuterium relaxation rates $R_{1}\left({ }^{2} \mathrm{H}\right)$ and self-diffusion coefficients $D_{\mathrm{w}}$ in these systems. The corresponding parameters in eq 11 have been tabulated in ref 10 and will not be repeated here.

Inserting the radius of the cations, i.e. assuming $R_{\text {eff }}=R_{+}=$ $0.347 \mathrm{~nm}$, into eq 10 gives corresponding relaxation rates which are too large by a factor of 3 , roughly whatever the value of the correlation time $\tau_{\text {int }}$ of the internal mode. On the other hand, if one ignores the theoretical order parameter $S^{2}\left({ }^{1} \mathrm{H}\right)=0.25$ for a moment and assumes that the fast methyl group reorientation provides the main relaxation path, i.e. set $S^{2}\left({ }^{1} \mathrm{H}\right)=0$, then the calculated relaxation rate curve fits the data at high temperatures but deviates strongly toward smaller relaxation rates at low temperatures with a calculated relaxation rate maximum which is smaller than the experimental relaxation rate at the lowest temperatures measured. Thereby the reasonable assumption has been made, that the thermally activated internal methyl group reorientation exhibits an Arrhenius temperature dependence with an apparent activation energy $E_{\mathrm{a}}=1450 \mathrm{~K}$ and a preexponential factor corresponding to a typical librational frequency, i.e. $\tau_{10} \approx$ $1 \times 10^{-14} \mathrm{~s}^{26}$ The value of the energy barrier $E_{\mathrm{a}} \approx 12 \mathrm{~kJ} / \mathrm{mol}$, as obtained from the fit, is within the range of barriers $12 \mathrm{~kJ} / \mathrm{mol}$ $\leq E_{\mathrm{a}} \leq 15 \mathrm{~kJ} / \mathrm{mol}$ reported in the literature in case of internal methyl group reorientations. ${ }^{24}$

As a consequence the isobaric temperature dependence of the proton and carbon-13 relaxation rates is, in the range of temperatures studied, largely dominated by the overall tumbling motion of the cations. Accounting for the non-Arrhenius temperature dependence of the experimental relaxation time curves $T_{1}(T, p, c), \tau_{+, \text {or }}(T, p, c)$ has been represented by

$$
\tau_{+, \mathrm{or}}(T, p, c)=\tau_{0} \exp \left(B_{+, \mathrm{or}}(p, c) /\left(T-T_{0}(p, c)\right)\right)
$$

with $T_{0}$ taken from ref 10 and $\tau_{0}$ and $B_{+, \mathrm{or}}(p, c)$ adjusted simultaneously in a nonlinear least squares fit to the experimental proton and carbon- 13 relaxation data. Note that only the slope
TABLE 3: Model Parameters To Calculate the Correlation Times of the Overall Tumbling $\tau_{+, \text {or }}$ and the Internal Methyl Group Reorientation $\tau_{\text {int }}$ of the Organic Cations $\mathrm{Me}_{4} \mathrm{~N}^{+}\left(\tau_{0}=\right.$ $\left.0.45 \pm 0.05 \mathrm{ps} ; \tau_{10}=5 \pm 3 \mathrm{fs} ; E_{\mathbf{2}}=1400 \pm 100 \mathrm{~K}\right)$

\begin{tabular}{lccccccc}
\hline & \multicolumn{7}{c}{$p(\mathrm{MPa})$} \\
\cline { 2 - 7 } & 0.1 & 5 & 50 & 100 & 150 & 225 \\
\hline$T_{0} \pm 3(\mathrm{~K})$ & 143 & 134 & 130 & 125 & 125 & 150 \\
$B_{+, \text {or }} \pm 10(\mathrm{~K})$ & 400 & 430 & 460 & 480 & 500 & 520 \\
& & $c=2.0 m$ & & & \\
$T_{0} \pm 3(\mathrm{~K})$ & 138 & 136 & 134 & 131 & 128 & 128 \\
$B_{+, \text {or }} \pm 10(\mathrm{~K})$ & 450 & 460 & 475 & 490 & 490 & 490 \\
& & $c=3.0 m$ & & & \\
$T_{0} \pm 3(\mathrm{~K})$ & 134 & 134 & 132 & 127 & 127 & 122 \\
$B_{+, \text {or }} \pm 10(\mathrm{~K})$ & 480 & 510 & 500 & 520 & 520 & 540
\end{tabular}

parameter $B_{+, 0 r}$ had to be changed with varying pressure and solute concentration. Although $\tau_{0}$ and $B_{+, \text {or }}$ are highly correlated, the latter can be determined rather reliably from the slope fo the experimental relaxation rates in a modified Arrhenius diagram $\ln T_{1}$ vs $1 /\left(T-T_{0}\right)$. The temperature dependence of the correlation time $\tau_{\text {int }}$ of the methyl group reorientation may be represented by an Arrhenius equation

$$
\tau_{\text {int }}(T, p, c)=\tau_{\text {io }} \exp \left(E_{\mathrm{a}} / k T\right)
$$

with $\tau_{\mathrm{io}}=5 \mathrm{fs}$ and $E_{\mathrm{a}}=1400 \mathrm{~K}$ chosen in accord with the results discussed above. If one calculates both correlation times at any given set of external variables it turns out that the overall tumbling mode is always at least an order of magnitude slower than the internal mode. This implies that $\tau \approx \tau_{\text {int }}$ in eq 5 to good approximation. Given the geometric weight $\left(S^{2}\right)$ on both motional modes entering the spectral density function, the methyl group reorientation does not contribute much to the relaxation of the protons and both modes contribute about aqually to the relaxation of the carbon- 13 nuclei in the region of the phase space investigated. As a result the parameters $\tau_{\text {io }}$ and $E_{\mathrm{a}}$ cannot be determined very reliably and were therefore kept constant throughout. The values of these parameters should not be taken too serious as long as there are no data at much lower temperatures as an infinite set of pairs $\left(\tau_{i o}, E_{\mathrm{a}}\right)$ would give similar results because of their strong correlation. However, the data are sensitive to the absolute magnitude of the correlation times $\tau_{\text {or }}$ and $\tau_{\text {int }}$ within the given motional model. Hence the general conclusions are valid irrespective of the set of parameters which solve the fitting exercise. It is noteworthy, then, that the correlation times $\tau_{\text {int }}$ for the internal methyl group reorientation turn out to be rather $\operatorname{short}\left(\tau_{\text {int }}(298 \mathrm{~K})\right.$ $<1 \mathrm{ps}$ ) compared to a typical value of $5 \mathrm{ps}$ appropriate to methyl groups attached to long alkyl chains and exposed to a typical liquid alkane environment. ${ }^{27}$ This seems to support the finding from recent computer simulations ${ }^{28}$ that the $\mathrm{Me}_{4} \mathrm{~N}^{+}$ion is surrounded by a rather substantial cavity formed by the nearest neighbor water molecules. Whether or not the aqueous matrix around the apolar surface of the $\mathrm{Me}_{4} \mathrm{~N}^{+}$cations allows for a less hindered methyl group reorientation remains an open question that will be considered further in a forthcoming paper ${ }^{29}$ on the molecular dynamics of the higher homologues of these symmetric tetraalkylammonium ions. All model parameters are compiled in Table 3, and the calculated relaxation times are shown as full lines drawn through the data in Figure 4a-c.

Also shown are the proton relaxation times of the solvent $\mathrm{HDO}$ molecules in the $5 \% / 95 \% \mathrm{D}_{2} \mathrm{O} / \mathrm{H}_{2} \mathrm{O}$ mixture. The relaxation of the related proton spin system is mediated almost exclusively by dipolar interactions with the methyl protons of the dissolved cations. Both relaxation time curves approach each other indicating, as is indeed observed with the higher homologues $\mathrm{Et}_{4} \mathrm{~N}^{+}, \mathrm{Pr}_{4} \mathrm{~N}^{+}$, and $\mathrm{Bu}_{4} \mathrm{~N}^{+}$as solutes, ${ }^{29}$ that at very low temperatures cross-relaxation between the hydration water protons and the methyl protons on the apolar surface of the solutes may dominate. This is illustrated in Figure 5, which compares 


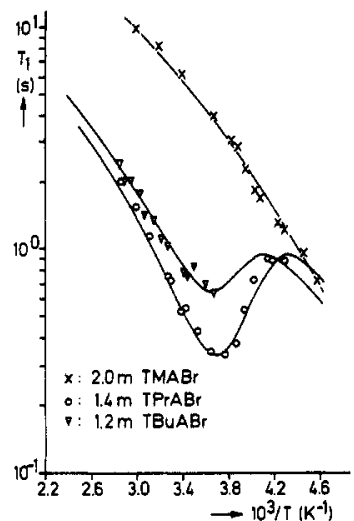

Figure 5. Comparison of the methyl proton spin-lattice relaxation times of the dissolved cations in undercooled aqueous solutions of $\mathrm{Me}_{4} \mathrm{~N}^{+}, \mathrm{Pr}_{4} \mathrm{~N}^{+}$ and $\mathrm{Bu}_{4} \mathrm{~N}^{+}$ions.

the proton relaxation times of the methyl groups of the dissolved cations in solutions where all water molecules are nearest neighbors to these cations. It becomes obvious that all relaxation times become almost equal at low temperatures, which may indicate that it is the methyl group reorientation which will provide the most efficient relaxation channel under these conditions. A detailed comparison of the proton and carbon- 13 relaxation data in these systems will be given in a forthcoming paper. ${ }^{29}$

\section{NDIS Experiments and Partial Pair Correlation Functions}

Diffraction experiments were made on three samples of deuterated $\mathrm{Me}_{4} \mathrm{NBr}$. Using isotope substitution neutron difference methods, a technique developed originally with respect to electrolyte solutions by Enderby and co-workers, ${ }^{30}$ one can effectively sit on an atom of interest in the aqueous solution and measure a partial pair correlation from this atom to its surroundings. Isotope substitution in the water then provides a means to determine water-water correlations through $g_{\mathrm{HH}}$ and $g_{\mathrm{HX}}$ pair correlation functions essentially, where $\mathrm{X}$ represents any nonsubstituted atom in the solution. Considering the system investigated, one can thus obtain direct information on the average structure of the water molecules coordinated to the apolar surface of the dissolved cations.

Data Analysis. The quantity obtained in a diffraction experiment after normalization and correction for multiple scattering and self-scattering is the total structure factor given by ${ }^{6}$

$$
F(Q)=\sum_{\alpha \beta} c_{\alpha} c_{\beta} b_{\alpha} b_{\beta}\left(S_{\alpha \beta}(Q)-1\right)
$$

where $c_{\alpha}$ is the atomic fraction of species $\alpha$ and $b_{\alpha}$ is its coherent scattering length. The partial structure factor $S_{\alpha \beta}(Q)$ of species $\alpha$ and $\beta$ is given by

$$
S_{\alpha \beta}(Q)-1=4 \pi \rho \int\left(g_{\alpha \beta}(r)-1\right)(\sin Q r / Q r) r^{2} \mathrm{~d} r
$$

with the atomic number density and $g_{\alpha \beta}(r)$ the partial pair correlation function of the atomic species $\alpha$ and $\beta$. If $H / D$ substitution in the water is used, the total structure factor from one solution is

$$
\begin{array}{r}
F(Q)=c_{\mathrm{H}}{ }^{2} b_{\mathrm{H}}{ }^{2}\left(S_{\mathrm{HH}}(Q)-1\right)+2 c_{\mathrm{H}} c_{\mathrm{X}} b_{\mathrm{H}} b_{\mathrm{X}}\left(S_{\mathrm{HX}}(Q)-1\right)+ \\
c_{\mathrm{X}}{ }^{2} b_{\mathrm{X}}{ }^{2}\left(S_{\mathrm{XX}}(Q)-1\right)
\end{array}
$$

and the three partial structure factors can then be obtained from a minimum of three solutions with contrasting values of $b_{\mathrm{H}}$, the average neutron scattering length of hydrogen in the solution. ${ }^{31}$ The $S_{\mathrm{HX}}$ and $S_{\mathrm{XX}}(Q)$ functions are actually weighted averages of several partial structure factors whereby

$$
\begin{gathered}
S_{\mathrm{HX}}(Q)=\sum_{\alpha \neq \mathrm{H}}\left(c_{\alpha} b_{\alpha} / c_{\mathrm{X}} b_{\mathrm{X}}\right) S_{\mathrm{H} \alpha}(Q) \\
S_{\mathrm{XX}}(Q)=\sum_{\alpha, \beta \neq \mathrm{H}}\left(\left(2-\delta_{\alpha \beta}\right) c_{\alpha} c_{\beta} b_{\alpha} b_{\beta} /\left(c_{\mathrm{X}} b_{\mathrm{X}}\right)^{2}\right) S_{\alpha \beta}(Q)
\end{gathered}
$$

$b_{\mathrm{X}}$ is the average scattering length of unlabeled atoms in the solution, given by

$$
b_{\mathrm{X}}=\sum_{\alpha \neq \mathrm{H}} c_{\alpha} b_{\alpha} / c_{\mathrm{X}}, \quad c_{\mathrm{X}}=\sum_{\alpha \neq \mathrm{H}} c_{\alpha}
$$

The partial pair correlation functions $g_{\alpha \beta}(\mathrm{r})$ are Fourier transforms of the corresponding partial structure factors. They were calculated by an iterative fitting routine designed to produce the minimum structure in the result compatible with the input data as described in detail in ref 13 . The goodness of the result was assessed on the basis of a quality factor defined as the weighted sum of (a) the value of the $\chi^{2}$ statistics corresponding to the agreement between the trial $S_{\alpha \beta}(Q)$ and the data and (b) a measure of the noise in the trial $g_{\alpha \beta}(r)$. An indicator of the systematic error in the data is provided by an integral over the first peak in each of the partial pair correlation functions which corresponds to a known intramolecular distance and coordination number.

The coordination number of atoms $\beta$ with atomic fraction $c_{\beta}$ in a spherical shell of thickness $\mathrm{d} r$ at a distance $r$ from any atom $\alpha$ is defined as

$$
\mathrm{d} N_{\alpha \beta}=4 \pi r^{2} \rho c_{\beta} g_{\alpha \beta}(r) \mathrm{d} r
$$

Hence the running coordination number, i.e. the average number of $\beta$ particles within a sphere of radius $r$ and $\alpha$ at the origin, is given by

$$
N_{\alpha \beta}(r)=4 \pi \rho c_{\beta} \int g_{\alpha \beta}(r)\left(r^{\prime}\right)^{2} \mathrm{~d} r^{\prime}=c_{\beta} n_{\alpha \beta}
$$

The coordination number of $H$ atoms given by the first intramolecular peak in $g_{\mathrm{HH}}(r)$, corresponding to the intramolecular $\mathrm{H}-\mathrm{H}$ distance at $0.155 \mathrm{~nm}$ in water, is thus

$$
N_{\mathrm{HH}}=4 \pi \rho c_{\mathrm{H}} \int g_{\mathrm{HH}}\left(r^{\prime}\right)(r)^{2} \mathrm{~d} r^{\prime}=c_{\mathrm{H}} n_{\mathrm{HH}}
$$

and must be equal to 1.0 . Similarly the first peak in the $H X$ correlation function corresponds to the intramolecular $\mathrm{H}-\mathrm{O}$ distance in the water molecule and the coordination number of oxygen atoms at a distance from any hydrogen is by definition

$$
N_{\mathrm{HO}}=\left(c_{\mathrm{X}} b_{\mathrm{X}} / b_{\mathrm{O}}\right) n_{\mathrm{HX}}
$$

which again must equal 1.0 .

Structure Factors and Pair Correlation Functions. Figure 6 shows the fits to the $S_{\mathrm{HH}}(Q)$ and $S_{\mathrm{XH}}(Q)$ resulting from the Fourier transform routine, for the room temperature $(298 \mathrm{~K})$ and the undercooled phase $(258 \mathrm{~K})$ data. The crosses show the input data after subtraction of a background function corresponding to unphysical features in the pair correlation functions. Figure 7 shows the resulting partial pair correlation functions $g_{\alpha \beta}(r)$. The room temperature results obtained from solutions contained in silica tubes agree within the error limits with results obtained with $\mathrm{TiZr}$ cells as sample containers. ${ }^{6}$ This provides further confidence in the reliability of the corrections applied to the raw data.

The general features of the $\mathrm{HH}$ correlation for the water in the solution are very similar to pure water, ${ }^{6}$ which is the same result as that found from computer simulations of related systems. ${ }^{32}$ The first peak in $g_{\mathrm{HH}}(r)$ corresponds to the intramolecular $\mathrm{HH}$ distance, and the coordination number should be equal to one $\mathrm{H}$ atom. The experimental coordination numbers obtained by integrating $g_{\mathrm{HH}}(r)$ up to the first minimum are 0.8 

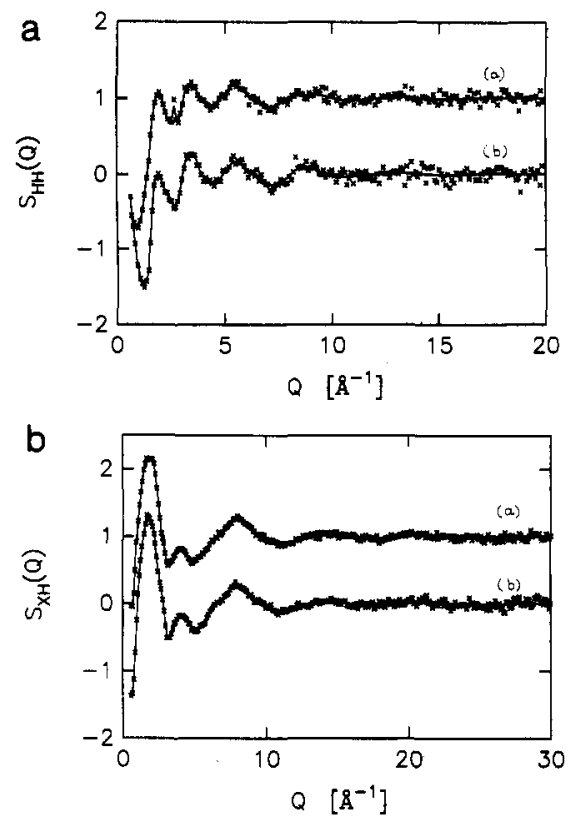

Figure 6. Partial structure factors $S_{\alpha \beta}(Q)$ in aqueous $\mathrm{Me}_{4} \mathrm{NBr}$ solutions at the temperatures (a) 298 and (b) $258 \mathrm{~K}$. To avoid data overlap curves $b$ have been shifted by one unit.
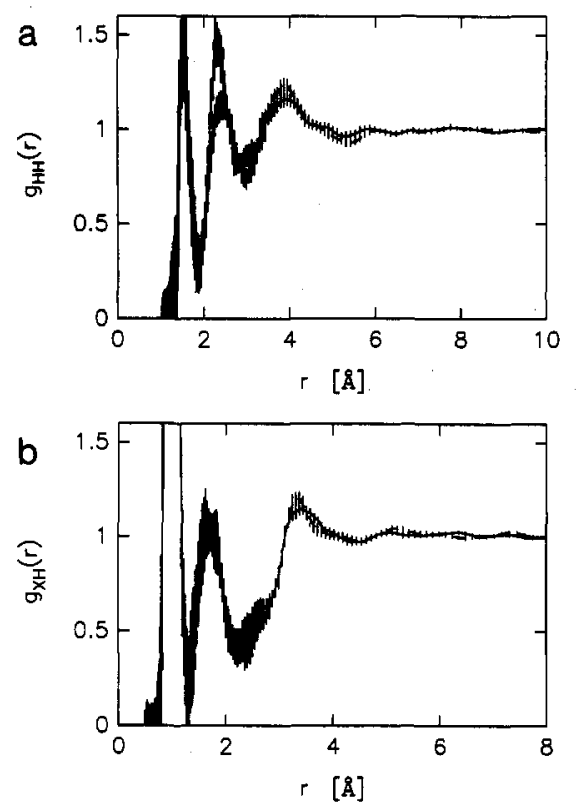

Figure 7. Partial pair correlation functions $g_{\alpha \beta}(r)$ in aqueous $\mathrm{Me}_{4} \mathrm{NBr}$ solutions at the temperatures (a) 298 and (b) $258 \mathrm{~K}$.

\pm 0.2 for the low-temperature result and $0.7 \pm 0.3$ for the room temperature data. Besides the intramolecular peak at $0.155 \mathrm{~nm}$, three intermolecular distances characterize the orientational structure of hydrogen bonded water at approximately $0.230,0.300$, and $0.380 \mathrm{~nm}$, as is sketched in Figure 8 . Because of disorder induced by thermal excitations in the $\mathrm{H}$-bond network of water, the first two distances cannot be resolved at room temperature. In the undercooled phase the thermal disorder is reduced strongly and there is clearly significant difference between the results for the two temperatures in the region of the second peak (the intermolecular first neighbor $\mathrm{HH}$ distance). For the $258 \mathrm{~K}$ data the peak is sharper, in the sense that it is higher and the position of the second minimum is moved in by $0.1 \AA$, although the minima are not in fact deeper. This may be because the reduction in thermal disorder enables the second neighbor (3.0- $\AA$ ) distance to be better resolved from the first neighbor distance at $258 \mathrm{~K}$. The position of the maximum of the second peak is also about $0.1 \AA$ shorter in the $258 \mathrm{~K}$ data. This distance is close to what would be expected from "ideal" tetrahedral hydrogen bonding in

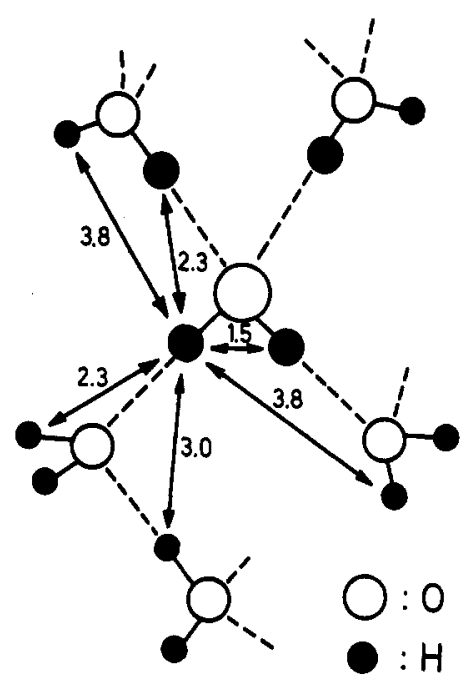

Figure 8. Schematic drawing of the nearest neighbor geometry in an ideal H-bond network of water.

water (i.e. with $\mathrm{OH}$ distances of 1.0 and $1.8 \AA$ and $\mathrm{HOH}$ angle of $110^{\circ}$ ). The room temperature data, on the other hand, has a second peak position of about $2.4 \AA$, which is closer to the value for pure water at room temperature. This suggests that the water in the $258 \mathrm{~K}$ solution may be closer to a fully hydrogen bonded network. The third peak position is the same at both temperatures (and in pure water at room temperature), although the peak may be somewhat sharper for the $258 \mathrm{~K}$ data, again suggesting an increase in order. The related coordination number is the same at both temperatures and amounts to $3.7 \pm 0.3$. This value is considerably lower than that found in room temperature water, where it is 5.5 roughly. If the water in the cold solution has more complete hydrogen bonding, some evidence of long-range correlations would be expected in $g_{\mathrm{HH}}(r)$. Although the region from about 4.5 to $6 \AA$ does show some structure for the $258 \mathrm{~K}$ data, there is not enough significant detail to say that the longer distance region definitely confirms the existence of enhanced hydrogen bonding, which the first and the second neighbor regions seem to indicate.

In pure water the first two peaks in $g_{\mathrm{HX}}(r)$ correspond to the intramolecular $\mathrm{H}-\mathrm{O}$ distance and the first neighbor $\mathrm{H} \ldots \mathrm{O}$ hydrogen bond distance. In the solution under investigation the coordination number of the first peak is $1.1 \pm 0.2$ as expected as this peak corresponds to the $\mathrm{H}-\mathrm{O}$ correlation only. It provides a further reference point indicating the reliability and internal consistency of the necessary corrections in the data treatment. The second peak corresponds closely to the $\mathrm{H}$... O distance in water but includes also minor contributions from $\mathrm{H}$... Br and $\mathrm{H}$...D(methyl) distances. Although the two correlation functions are very similar within the error bars, there is some indication that the second minimum is deeper and the third peak is sharper in the case of the $258 \mathrm{~K}$ data, indicating a reduction in thermal motion or an increase in hydrogen bonding. The observations are in fact similar to trends observed in computer simulations of undercooled liquid water, ${ }^{33,34}$ amorphous water clusters, ${ }^{35}$ and aqueous solutions of apolar solutes. ${ }^{32}$

A possible interpretation of the low-temperature $(258 \mathrm{~K})$ results is that the first neighbor structure in the hydration water is close to the ideal hydrogen bonded case, with four neighbors up to a distance of $0.230 \mathrm{~nm}$ and one neighbor at around $0.300 \mathrm{~nm}$. Since the sharpness of the peaks in the $\mathrm{HH}$-correlation function is a direct measure of the degree of orientational correlations between neighboring water molecules, the narrower first peak at low temperature seen here suggests that below ambient temperatures the orientational correlation between molecules becomes strongly dependent on temperature. By contrast the same peak in the room temperature result is broader and the minimum is at a higher value of $r$, indicating greater structural disorder. The structure between 0.300 and $0.600 \mathrm{~nm}$ is also significantly sharper, 
suggesting less disorder in the H-bond network. The low coordination number of about 4 compared to about 5 in pure water at room temperature shows that the number of nearest neighbors is reduced in the hydration shell of the apolar solute relative to the $\mathrm{H}$-bond network of pure water. This effect has been found also in $\mathrm{Me}_{4} \mathrm{NCl}$ solutions at room temperature. ${ }^{28,29}$ On the other hand the hydrogen bonding geometry of pure water is essentially retained as far as the first and second neighbors are concerned. There seems to be no indication for any longer range ordering in the hydration water induced by the apolar surface despite the significantly enhanced structural features observed in the undercooled phase. As no partial pair correlation functions $g_{\alpha \beta}(r)$ of pure undercooled water are available in the literature, any direct comparison with the present $g_{\mathrm{HH}}(r)$ must await their experimental determination.

\section{Conclusions}

Multinuclear spin-lattice relaxation rates and self-diffusion coefficients of both solvent and solute molecules have been reported over large ranges of temperature, pressure, and concentration in undercooled aqueous solutions of $\mathrm{Me}_{4} \mathrm{~N}^{+}$ions. The distortion of the random, transient $\mathrm{H}$-bond network of liquid water by the dissolved cations was shown to lead to complex changes of the dynamics of the solvent molecules coordinated to the apolar ions. Because of a subtle balance of Coulombic, hydrophobic hydration, and $\mathrm{H}$-bond interactions, the changes in solvent mobility relative to pure water, i.e. retardation at high temperatures but facilitation at low temperatures of both rotational and translational motions, depend strongly on the state of the $\mathrm{H}$-bond network of the water of hydration. These dynamical features are incompatible with a frequently suggested clathrate-like organization of the water molecules for which the term hydrophobic hydration has been coined in the literature. The translational mobility of the cations corroborates this conclusion in that it reveals a highly transient and short-lived hydration cage even in the undercooled phase. Their overall tumbling motion is also faster than that predicted by simple hydrodynamic theory. The rather fast internal methyl group reorientation supports the finding from computer simulations that dissolved $\mathrm{Me}_{4} \mathrm{~N}^{+}$ions are surrounded by a substantial cavity formed by the coordinated water molecules. Hence sterical hindrance of the methyl group reorientation by the solvent molecules may not be expected. This remains valid in the undercooled phase also, as the proton relaxation times of the solvent and solute molecules become inreasingly similar at low temperatures, signifying the onset of strong cross-relaxation with the fast methyl group rotation providing a relaxation sink for the whole system. These dynamical features have been confronted with structural information deduced from neutron diffraction difference experiments. Using $H / D$ isotope substitution in the solvent, all water-water correlations have been obtained for the first time in undercooled aqueous solutions of $\mathrm{Me}_{4} \mathrm{~N}^{+}$ions. The composition of the solution renders all water molecules nearest neighbors to the cations. Compared to room temperature hydration water, a significant difference in the sharpness and position of the intermolecular peaks in $g_{\mathrm{HH}}$ was obtained showing a reduction in disorder and possibly a tendency to a more "ideal" hydrogen bonding geometry. It is interesting in this respect that the positional and orientational correlations within the hydration shell do not seem to be vastly different from the bulk water phase at room temperature, although the NMR data show a considerable difference in the respective dynamic properties. Though the present NDIS data indicate an enhanced order in the transient
$\mathrm{H}$-bond network of cold water, a direct comparison with related structural correlations in pure water must await the determination of the corresponding partial structure factors in undercooled water. In summary, measurements in the undercooled phase where thermal distortions are strongly reduced may prove useful in the future to help identify the structural and dynamical characteristics of the so-called hydrophobic hydration of apolar solutes.

Acknowledgment. The experiments would not have been possible without the expert technical help of R. Knott, D. Niesner, S. Heyn, and E. Treml. We further thank Prof. Lüdemann for providing access to the MSL 300 NMR spectrometer. Financial support of the NMR experiments by the DFG is gratefully acknowledged. The NDIS experiments have been done at the ISIS neutron facility of the Rutherford Appleton Laboratory. Financial support of these experiments by the SERC is gratefully acknowledged also.

\section{References and Notes}

(1) Enderby, J. E.; Cummings, S.; Herdman, G. J.; Neilson, G. W.; Salmon, P. S.; Skipper, N. J. Phys. Chem. 1987, 91, 5851 .

(2) Lang, E. W.; Fink, W.; Radkowitsch, H.; Girlich, D. Ber, BunsenGes. Phys. Chem. 1990, 94, 342.

(3) Wen, W.-Y. In Water and Aqueous Solutions; Horne, R. A., Ed.; Wiley: New York, 1972; pp 613-661.

(4) Huot, J.-Y.; Jolicoeur, C. In The Chemical Physics of Solvation; Dogonadze, R., Kalman, E., Kornyshev, A. A., Ulstrup, J., Eds.; Elsevier: Amsterdam, 1985; pp 417-471.

(5) Finney, J.; Turner, J. Faraday Discuss. Chem. Soc. 1988, 85, 125.

(6) Turner, J.; Soper, A. K.; Finney, J. Mol. Phys. 1990, 70, 679; 1992, $77,411,431$

(7) Guillot, B.; Guissani, Y.; Bratos, S. J. Chem. Phys. 1991, 95, 3643. 263.

(8) Geiger, A.; Rahman, A.; Stillinger, F. H. J. Chem. Phys. 1979, 70,

(9) Stillinger, F. H. Science 1980, 209, 451.

(10) Bradl, S.; Lang, E. W. J. Phys. Chem. 1993, 97, 10463.

(11) Lang, E. W.; Lüdemann, H.-D. In NMR-Basic Principles and Progress; Jonas, J., Ed.; Springer: Berlin, 1990; Vol. 25, pp 131-187.

(12) Lang, E. W.; Lüdemann, H.-D. In Progress in NMR Spectroscopy: Emsley, J. W., Feeney, J., Sutcliff, L. H., Eds.; 1993; Vol. 25, pp 507-634.

(13) Soper, A. K. In Neutron Scattering Data Analysis; Johnson, M. W., Ed.; IOP: Bristol, 1990; Vol. 107, p 57.

(14) Soper, A. K.; Howells, W. S.; Hannon, A. C. Rutherford Appleton Laboratory Report; Rutherford Appleton Laboratory: Oxon, U.K., 1989, p $46 \mathrm{ff}$.

(15) Eriksson, P.-O.; Lindblom, G.; Burnell, E. E.; Tiddy, G. J. T. J. Chem. Soc., Faraday Trans. 1 1988, 84, 3129.

(16) Hertz, H. G.; Lindman, B.; Siepe, V. Ber. Bunsen-Ges. Phys. Chem. $1969,73,542$.

(17) Easteal, A. J.; Woolf, L. A. J. Solution Chem. 1986, 15, 1003.

(18) Hertz, H. G.; Holz, M. J. Phys. Chem. 1974, 78, 1002.

(19) Spiess, H. W. In NMR-Basic Principles and Progress; Diehl, P., Fluck, E., Kosfeld, R., Eds.; Springer: Berlin, 1978; Vol. 15, p $55 \mathrm{ff}$.

(20) Lipary, G.; Szabo, A. J. Am. Chem. Soc. 1982, 104, 4546, 4559.

(21) Halle, B.; Wennerström, H. J. Chem. Phys. 1981, 75, 1928.

(22) Kratky, O.; Leopold, H.; Stabinger, H. In Methods in Enzymology; Hirs, C. H. W., Timasheff, S. N., Eds.; Academic Press: New York, 1973; p 27.

(23) Holz, M.; Patil, K. J. Ber. Bunsen-Ges. Phys. Chem. 1991, 95, 107. (24) Lang, E. W.; Bradl, S.; Kunz, W.; Turq, P. J. Phys. Chem. 1991, 95 , 10576

(25) Kivelson, D. Discuss. Faraday Soc. 1977, 117

(26) Brown, A. N.; Newbery, M.; Thomas, R. K.; White, J. W. J. Chem. Soc., Faraday Trans. 2 1988, 84, 17.

(27) Bratt, P. J.; Gilles, D. G.; Sutcliffe, L. H.; Williams, A. J. J. Phys. Chem. 1990, 94, 2727.

(28) Jorgensen, W.; Gao, J. J. Phys. Chem. 1986, 90, 2174.

(29) Bradl, S.; Lang, E. W. Manuscript in preparation.

(30) Enderby, J. E.; Neilson, G. W. In Water-A Comprehensive Treatise;

Franks, F., Ed.; Plenum Press: New York, 1973; Vol. 6, pp 1-46.

(31) Soper, A. K.; Phillips, M. C. Chem. Phys. 1986, 107, 47.

(32) Alagona, G.; Tani, A. J. Chem. Phys. 1980, 72, 580.

(33) Jorgensen, W. J. Chem. Phys. 1982, 77, 4156.

(34) Jorgensen, W. J. Chem. Phys. 1983, 79, 926.

(35) Zhang, Q.; Buch, V. J. Chem. Phys. 1990, 92, 5004. 